\title{
Rainfall Prediction using Back-Propagation Feed Forward Network
}

\author{
Ankit Chaturvedi \\ Department of CSE \\ DITMR (Faridabad) \\ MDU Rohtak (hry).
}

\begin{abstract}
Back propagation is most widely used in neural network projects because it is easy to train and for its accuracy. Back propagation learning algorithm consists of two facets, the first one generate the input pattern of the network and the another one to adjust the output through altering the weights of the network. The back propagation algorithm can be for predicting rainfall. This paper materialize training, testing of data set and detecting the hidden neuron in the network. In this research, rainfall prediction in the region of DELHI (India) has been analyzed using neural network back propagation algorithm. Three layer model has been used for training and studying different attributes of the hidden neurons in the network.
\end{abstract}

\section{Keywords}

Back-propagation, Artificial neural network, Prediction, Rainfall, Feed forward network.

\section{INTRODUCTION}

India is the country in which agriculture is the backbone of the country. Agriculture is the integral part of the economy which plays a vital role in the growth of economy. Rainfall prediction is the most important tool for growth of agriculture in the country. Rainfall prediction is most complicated and difficult procedure to perform accurately. Prediction needs large amount of archival and actual data from past records.

Rainfall plays very important role in human life and agriculture, it is very essential for irrigation. Rainfall prediction is also useful for sewer management, water management and flood forecasting. Many factors influence rainfall prediction such as temperature, humidity, wind speed, pressure, dew point etc.

Artificial neural network can be used for predicting because of having the capability of examining and determining the historical data used for prediction. ANN has better accuracy than statistical and mathematical models. ANN works on the principle of biological neurons which is a type of data driven technique. The conjunction between meteorological parameters and rainfall can be analyzed using ANN.

Back propagation is the common method for training ANN. It is the supervised learning method which requires a dataset of the desired output from many inputs, making up the training set. It is most useful for feed-forward network (networks that have no feedback or simply, that have no connection in that loop).
The paper aims to develop ANN using back propagation for predicting the rainfall in Delhi region of India. The Indian economy highly depends on monsoon and seasonal rainfall so it is necessary and challenging or meteorological researchers to predict rainfall in different regions of the country.

\section{METHODOLOGY}

Methodology includes initial step of data mining. Data is collected from different meteorological websites, input and target data are decided for the prediction, database is created accordingly. For input and target previous data has been taken for prediction. The input and target data have to be normalized because of having different units. For normalization the mean of all the data i.e. humidity, wind speed and rainfall is calculated.

Let mean be M,

\section{$M=$ Sum of entries/number of entries}

Standard deviation (SD) of each entry is calculated. After calculating the mean and standard deviation the value of each parameter is normalized.

\section{Normalized value $=(\mathrm{x}-\mathrm{M}) / \mathrm{SD}$}

Normalization will preserve the relationship among the actual data values.

\subsection{ANN Architecture}

Artificial neural network is a physical cellular network that is able to acquire, store and utilize experimental knowledge which has been related to the network capability and performance. There are different types of neural networks and the one implemented in this paper is feed forward network

\subsubsection{Feed Forward Network}

In the generic feed forward network characterized by the lack of feedback. The information moves in only one direction that is forward, this type of network can be connected in cascade to create multilayer network. The layer consists of neurons and makes independent computation on data that it receives and passes to another layer. Weights are the connection between the two layers, neurons makes computation based upon weighted sum of inputs. The first layer of feed forward network is the input layer and the last layer is the output, other layer between the two layers are called hidden layers. A function called the threshold is used for calculating the output of the neuron in the output layer. 


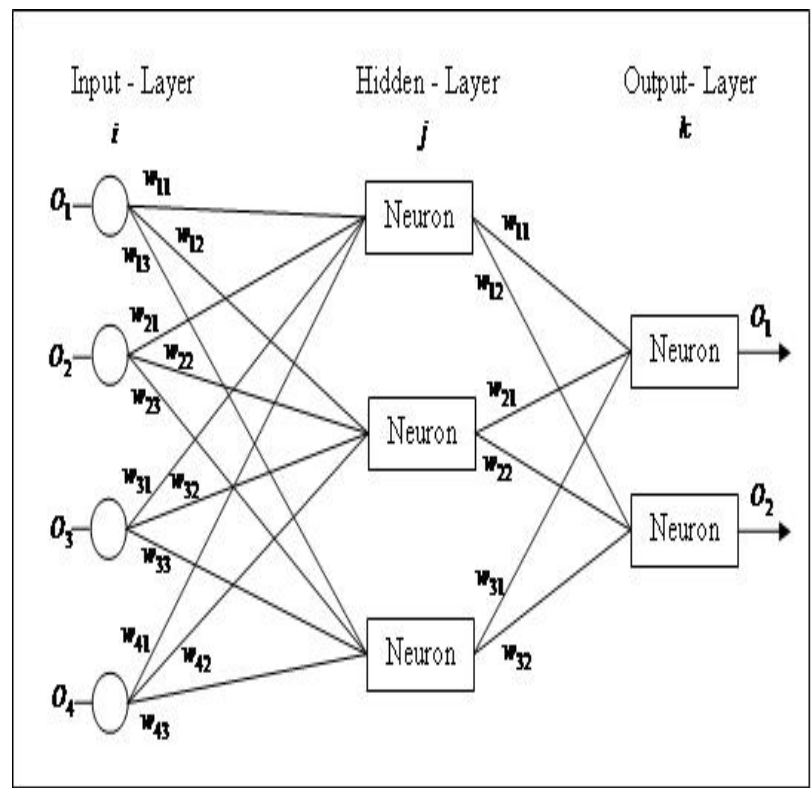

Fig 1. Feed Forward Network

\subsubsection{Selection of input and target data for prediction}

In this paper the input and target data is calculated for Delhi (India). The database is collected from different meteorological websites. Rainfall can be of two types seasonal and non- seasonal, seasonal rainfall occur during monsoon period that is from the month of May to the month of September and rest of the months causes non seasonal rainfall. It is very difficult to predict daily base data because it is tough to maintain accuracy in data and the parameters used for rainfall prediction, these parameters must be accurate for generating minimum mean square error. It is not easy to maintain accuracy of the parameters used for calculating daily basis data which can lead to high error in data training, testing and validation. The data is retrieved from IMD website.

\subsubsection{Training, Testing and Validation}

After creating the database and normalizing the data, next step is training of the input data using back propagation algorithm. Training of the input data in the MATLAB is done using NFTOOL and NNTOOL. Back propagation algorithm takes 70 percent of data for training, 15 percent for testing and 15 percent for validation. The input data consist of 365 samples out of which 255 samples are for training, 55 samples for testing and rest samples for validation.

\section{RESULTS AND DISCUSSION}

For getting different results MATLAB tools used are NFTOOL and NNTOOL and are described below.

NFTOOL(Neural network fitting tool): It is used for solving data fitting problems and consists of a two layer feed forward network trained with Levenberg- marquardt.

NNTOOL(Open network/Data manager): It allows to import, create, use and export neural network and data.

\subsection{Comparison of actual and predicted data}

The graph has been plotted to show clearly the two data i.e. actual and predicted. It shows the comparison between values of actual and predicted data as shown in the figure 2 .

Plotting a graph is the perfect way to make comparison between two data. The graph is plotted after testing and validation part is over. The graph can be plotted only by saving data in the work space.

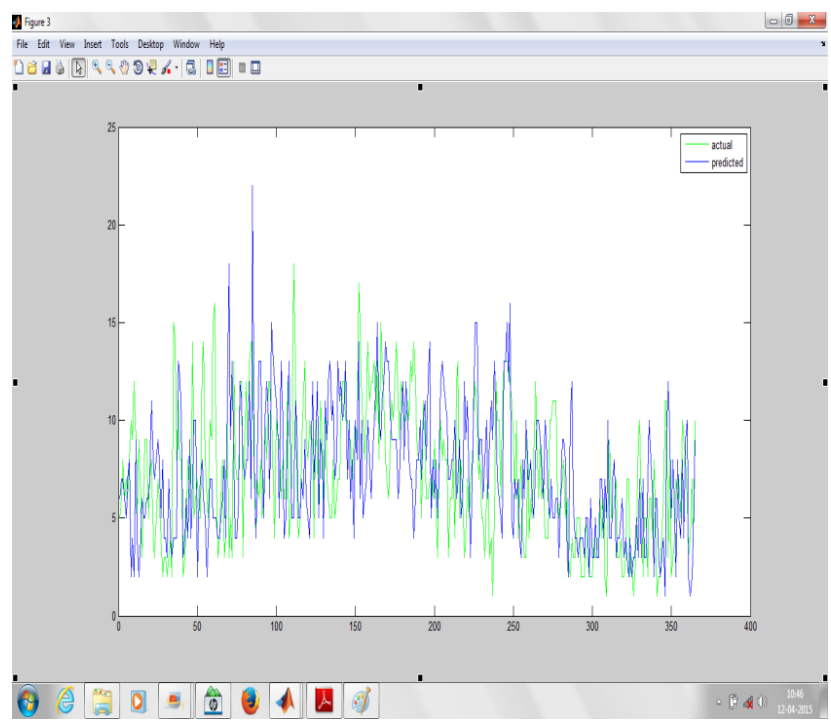

Fig 2.Snapshot of comparison between actual and predicted data

\subsubsection{Implementation of Back Propagation using NFTOOL}

The graph plotted using NFTOOL between predicted and target values show minimized MSE.

$$
\text { MSE }=8.70
$$

The performance graph can be plotted as in the figure 3 .

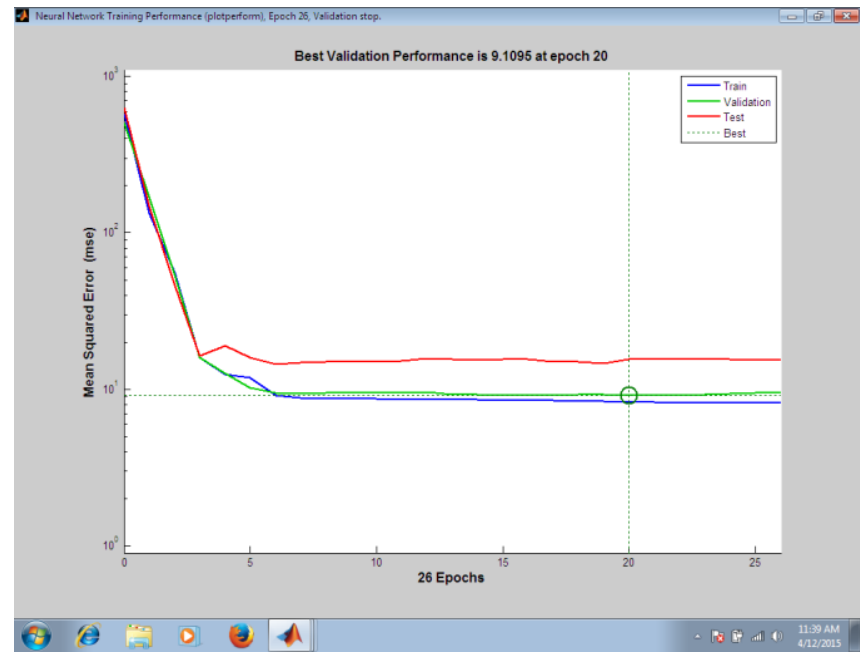

Fig 3.Snapshot of performance graph using NFTOOL 
The regression can be plotted as in figure 4 .

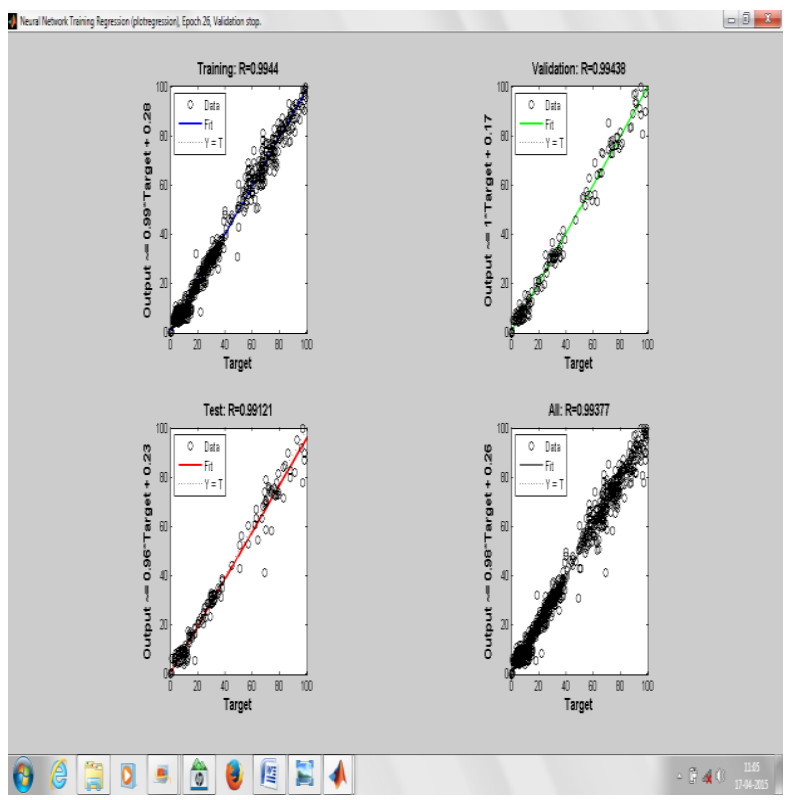

Fig. 4 Snapshot of Regression using NFTOOL

The above graph shows results using NFTOOL. The MSE observed was under tolerable level. It has high accuracy and minimized error,

\subsubsection{NNTOOL used for implementing multilayer architecture.}

Different algorithms were tested for multilayer architecture.

1) Back-propagation algorithm

2) Cascaded Back-propagation

3) Layer Recurrent network

Multilayer architecture tested with Back-propagation algorithm showed good consistency and accuracy. Two hidden layer were tested with 10-20 neurons in the multilayer architecture.

Back-propagation algorithm tested with sample data using different training function and adaptive learning function gave different results. The result with minimum error is shown in the figure 5 .

Table 1 Back-propagation testing cases

\begin{tabular}{|l|l|l|l|l|}
\hline S. No & $\begin{array}{l}\text { Training } \\
\text { Function }\end{array}$ & $\begin{array}{l}\text { Adaptive } \\
\text { learning } \\
\text { function }\end{array}$ & $\begin{array}{l}\text { No Of } \\
\text { neurons }\end{array}$ & MSE \\
\hline Case1 & TRAINLM & Learngdm & $\mathbf{1 0}$ & $\mathbf{6 . 9 7}$ \\
\hline Case2 & TRAINLM & Learngd & 10 & 10.50 \\
\hline Case3 & TRAINLM & Learngdm & 20 & 7.09 \\
\hline Case4 & TRAINLM & Learngd & 20 & 7.36 \\
\hline Case5 & TRAINRP & Learngdm & 10 & 9.90 \\
\hline Case6 & TRAINRP & Learngd & 10 & 9.57 \\
\hline Case7 & TRAINRP & Learngdm & 20 & 9.40 \\
\hline Case8 & TRAINRP & Learngd & 20 & 9.54 \\
\hline
\end{tabular}

In Table 1, case 1 has minimum MSE so it is the best case of back-propagation. The performance graph can be plotted as in figure number 5 .

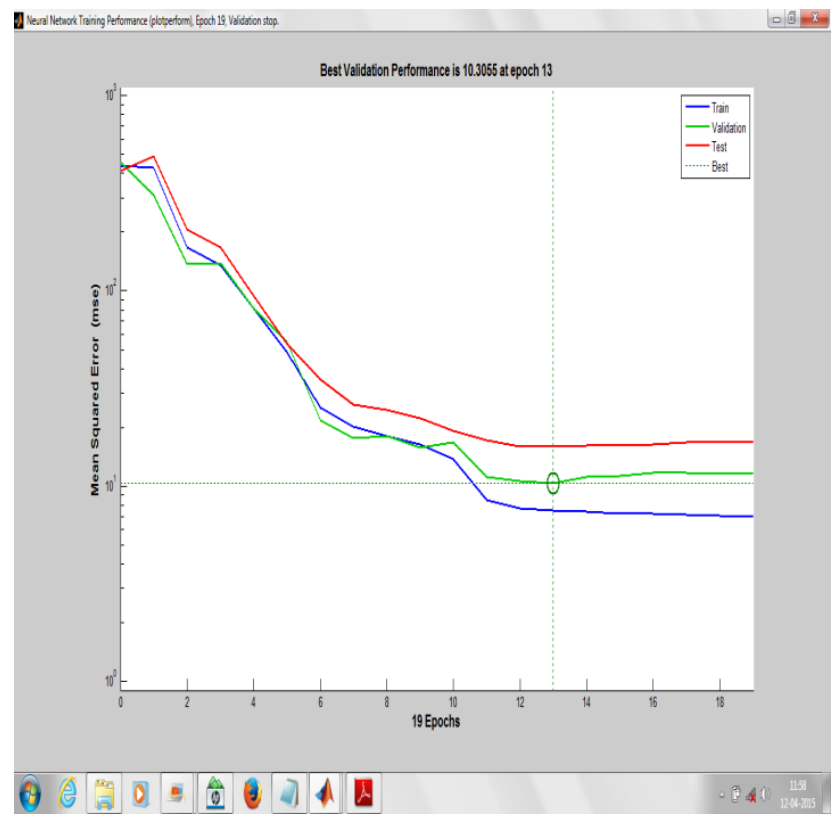

Fig.5 Back-propagation performance graph for case1

The graph plotted for the performance shows MSE and epochs. The graph clearly shows the best performance at 13 epochs. The training, test and validation is plotted in the performance graph.

Cascaded Back-propagation tested with sample data using different training function and adaptive learning function gave different results. The result with minimum error is shown in figure 6 .

Table 2 Cascaded back-propagation testing cases

\begin{tabular}{|l|l|l|l|l|}
\hline S.NO & $\begin{array}{l}\text { Training } \\
\text { Function }\end{array}$ & $\begin{array}{l}\text { Adaptive } \\
\text { Learning } \\
\text { function }\end{array}$ & $\begin{array}{l}\text { No. Of } \\
\text { neurons }\end{array}$ & $\begin{array}{l}\text { MS } \\
\mathrm{E}\end{array}$ \\
\hline Case1 & TRAINLM & Learngdm & 10 & 7.93 \\
\hline Case2 & TRAINLM & Learngd & 10 & 8.58 \\
\hline Case3 & TRAINLM & Learngdm & $\mathbf{2 0}$ & $\mathbf{7 . 6 5}$ \\
\hline Case4 & TRAINLM & Learngd & 20 & 7.91 \\
\hline Case5 & TRAINRP & Learngdm & 10 & 9.97 \\
\hline Case6 & TRAINRP & Learngd & 10 & 9.93 \\
\hline Case7 & TRAINRP & Learngdm & 20 & 9.30 \\
\hline Case8 & TRAINRP & Learngd & 20 & 9.54 \\
\hline
\end{tabular}

In Table 2, case 3 has minimum MSE, so it is the best case of cascaded back-propagation. The performance graph can be plotted as in figure number 6 .

Performance graph for cascaded back-propagation shown in figure 6 clearly shows the validation, training and testing for the best case that is case 3 in Table 2 . The best performance can be observed at epoch 4 .

In the Table 3, case 4 has minimum MSE, so it is the best case of layer recurrent network. 
Layer Recurrent network shows different results than the other two networks when tested with same training function and adaptive learning function. The result with minimum error is shown in figure 7.

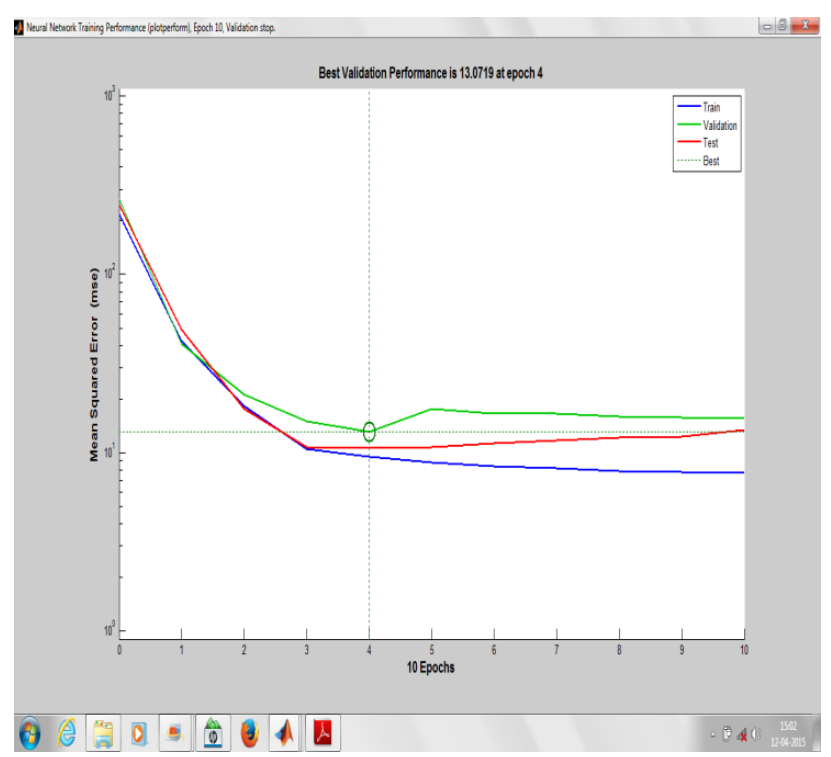

Fig 6 Casecaded back-propagation performance graph.

Table 3 Layer Recurrent testing cases.

\begin{tabular}{|l|l|l|l|l|}
\hline S.NO & $\begin{array}{l}\text { Training } \\
\text { function }\end{array}$ & $\begin{array}{l}\text { Adaptive } \\
\text { Learning } \\
\text { function }\end{array}$ & $\begin{array}{l}\text { No. } \\
\text { Of } \\
\text { neuro } \\
\text { ns }\end{array}$ & MSE \\
\hline Case1 & TRAINLM & Learngdm & 10 & 8.60 \\
\hline Case2 & TRAINLM & Learngd & 10 & 8.93 \\
\hline Case3 & TRAINLM & Learngdm & 20 & 9.54 \\
\hline Case4 & TRAINLM & Learngd & $\mathbf{2 0}$ & $\mathbf{8 . 2 2}$ \\
\hline Case5 & TRAINRP & Learngdm & 10 & 10.7 \\
\hline Case6 & TRAINRP & Learngd & 10 & 9.72 \\
\hline Case7 & TRAINRP & Learngdm & 20 & 9.53 \\
\hline Case8 & TRAINRP & Learngd & 20 & 9.67 \\
\hline
\end{tabular}

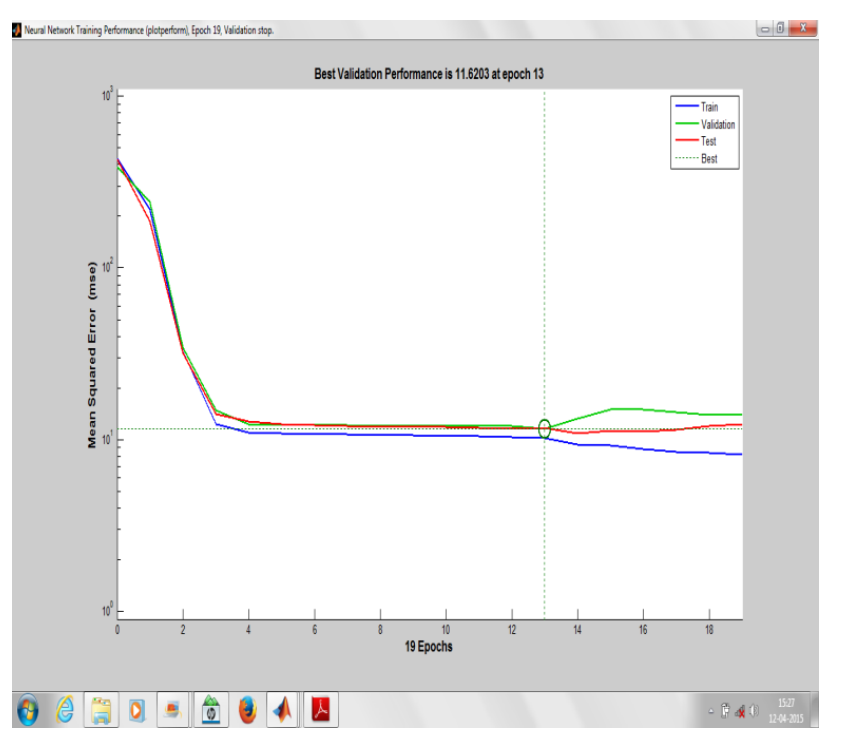

Fig 7 Layer Recurrent Network Performance graph
The graph plotted in figure 7 shows the performance for training, testing and validation. The best performance was observed in case 4 of layer recurrent network which has minimum MSE. Best performance is observed at epoch 13.

\section{CONCLUSIONS}

- Different networks give different results with same training functions and adaptive learning functions having same number of neurons.

- $\quad$ Back-propagation shows the best result out of the three networks.

- Increase in number of neurons of the network shows decrease in MSE.

- $\quad$ LEARNGDM observed to be the best adaptive learning function as it shows results with minimum MSE.

- $\quad$ TRAINLM showed best results in training, testing and validation of data.

- If the data is increased the performance of the network increases.

- NNTOOL is best for training the data using other algorithms and functions.

\section{ACKNOWLEDGEMENT}

The authors are thankful to Indian Meteorological Department for giving the rainfall and meteorological data of the work made.

\section{REFERENCES}

[1] Enireddy Vamsidhar, K.V.S.R.P.Verma P.Sankara Rao, Ravikanth Satapati "Prediction of Rainfall using Backpropagation neural network model", International Journal on Computer Science and Engineering Vol. 02, No. 04, 2010, 1119-1121.

[2] G.Geetha, R.Samuel Selvaraj "Prediction of Monthly Rainfall in Chennai using Back-propagation neural network model", International Journal of Engineering Science and Technology Vol. 3, No. 1, January 2011, 0975-5462.

[3] A.R Naik, S. K.Pathan, "Indian Monsoon Rainfall classification and prediction using robust backpropagation artificial neural network", International Journal of emerging technology and advanced engineering Vol. 3, No. 11, November 2013, 2250-2459.

[4] Santosh kumar Nanda Debi Prasad Tripathy, Simanta Kumar Nayak, Subhasis Mohapatra, "Prediction of Rainfall in India using Artificial neural network models", International Journal of intelligent systems and applications, November 2013, 1-22.

[5] V.K. Somvanshi, O.P.Pandey, P.K Agrawal, N.V Kalanker, M. Ravi Prakash, Ramesh Chand "Modelling and prediction of Rainfall using artificial neural network and ARIMA techniques", Journal of Indian and geophysical union Vol. 10, No. 2, April 2006, 141-151.

[6] Rohit R. Deshpandey, "On the rainfall time series prediction using multilayer perceptron artificial neural network", International Journal of emerging technology and advanced engineering Vol. 2, No. 1, January 2012, 2250-2459. 
[7] Prince Gupta, Satanand Mishra, S.K.Pandey "Time series data mining in rainfall forecasting using artificial neural network", International Journal of scientific engineering and technology Vol. 3, No. 8, August 2014, 1060-1065.

[8] Imran Maqsood, Muhammad Riaz Khan, Ajith Abraham "An ensemble of neural network for weather forecasting”, Neural Networks and applications, 2004, 112-122.

[9] S.Lee,S.Cho and P.M.Wong 1998,"Rainfall prediction using Artificial neural networks". journal of geographic information and Decision Analysis, Vol .2,No2,pp.233242.

[10] Stuart Russell and Peter Norvig, Artificial Intelligence A Modern Approach. p.578. "The most popular method for learning in multilayer networks is called Backpropagation. It was first invented in 1969 by Bryson and Ho, but was more or less ignored until the mid1980’s
[11] J. Thielen, B. Boudevllain, and H. Andrieu, "A radar data based short-tem rainfall prediction model" for urban areas Journal of Hydrology, vol. 239, 97-114, 2000. R. E. Tuleya,

[12] M. Demaria, and R. J. Kuligowski, "Evaluation of GFDL and simple statistical model rainfall forecasts for U.S. landfalling tropical storms", Weather and Forecasting, vol. 22, 56-70, 2007.

[13] K.N Mehta et.al., "Rainfall classification and prediction using back-propagation artificial neural network", International Journal of emerging technology and advanced engineering Vol. 3, No. 11, November 2013, 2250-2459.

[14] Jang, J.S.R., Sun, C.T. and Mizutani, E., Neuro-fuzzy and Soft Computing (A Computational Approach to Learning and Machine Intelligence), Prentice Hall, Inc., pp 1- 90, 1997

[15] Padhy, N.P., Artificial Intelligence and Intelligent systems, Oxford University Press, 2006. 